\title{
A Survey on Thangka Image Inpainting Method Based on Structure-borne
}

\author{
Xiaojing Liu \\ Department of Computer Technology and Application Qinghai University, Xining, China \\ 645020710@qq.com
}

Keywords: Thangka; structure-borne; image inpainting

Abstract. The ancient Tangka image with reflecting Tibet art has been broken by the nature and human with different degrees. The part-shed Tangka image is researched as a type of special breaking. After discussing digital image inpainting, the digital image restoration based on the structure, and Thangka image inpainting method, we give some direction of the development of Tangka image restoration based on structure-borne.

\section{Introduction}

Tangka, Tibetan word meaning "painted scroll", is an art of painting on silk or cloth. It is an integral part of Tibetan painting art with a long history. It is the important heritage of the Tibetan people for thousands of years. Its content involves interesting and colorful stories, relates to the history event, religion, personage, local conditions and customs, folklore, fairy story, building layout, astronomy calendar, Tibetan medicine, Tibetan pharmacology and so on. Tangka is the spirit of Buddhism. It is the "encyclopedia" of the world of technology and personal creativity.

However, the internal structure of some Tangka constantly changes because of ancient ages, temperature, humidity, light, radiation, harmful gases and dust, and many other natural and human factors. There is color flaking, fuzzy, black material, such as discoloration and so on in Tangka.

Most Thangka repair is redrawn by manual repair way, this way of repair not only big workload, and cannot be mass to repair damaged thangka, especially for repair of ancient Thangka, probably because of the mistaken understanding form error repair. The diversity of Thangka damage caused the thangka image damaged areas in the form of diversification. In number, and even may be a Thangka image in the existence of thousands of damaged area. Therefore, the work of digital preservation and repair of Tangka is very necessary and urgent. Thangka image, while complex, has certain forms of composition. How to use the method of computer aided and according to the structure characteristics of the Thangka itself, the damage form of Thangka and the extent of damage is analyzed, and virtual repair damage area of the image, has become the important research problem in the world of image restoration.

\section{Digital image inpainting}

Digital image restoration technique is lost or damaged part of the pointer to the image, according to its surroundings is not damage the effective information, according to certain rules to its fill, and the repaired image close to or achieve the visual effect of the original image. Image inpainting technology has a long history, originated in the 20s of the last century, is an ancient art of European Renaissance, at first is to restore lost or damaged part of the works of fine art, while keeping the overall effect of work, by hand painted to fill on cracks occurred in the fine art, make pictures get renovation. The craft technique is called "Retouching" or "Inpainting"(repair or repair). Until the late 1960s, with the constant innovation of computing technology, the digital image restoration technology got rapid development.

After more than 50 years of development, the technology of digital image restoration has become one of the current computer graphics and computer vision research hot spot, in the protection of cultural relics, the production of film and television special effects, virtual reality and so on has important application value. Through the constant pursuit of scholars at home and abroad and hard 
work, appeared in the field of image processing, a variety of digital image restoration model and algorithm. The model and algorithm combines not only a large number of digital signal processing, math, detection and estimation and colorimetry of relevant theoretical knowledge, but also make full use of the artificial intelligence, neural network and genetic algorithm.

General image is composed of two parts, one part is the structure of the image itself, the other part is the texture image. The basic framework of the structure of the image reflects the image itself and outline. This part of the information is a change in the overall image a reaction, and has a significant impact on human cognition image information. Accordingly, the image texture part bearing the image of local details information, and is detailed representation of the image information. At present, many domestic and foreign research scholars in the field of digital image restoration proposed a variety of effective restoration model and algorithm. One of the most typical two, respectively is based on the structure of the image restoration technology and based on the texture of image restoration technology. Based on Partial Differential equations image restoration algorithm is proposed based on the structure of image restoration technology, the typical algorithms including BSCB (Bertalmio Sapiro Caselles Ballester) model and the CDD (Curvature Driven Diffusions) model and later including the variational (Total Variation, TV) model ${ }^{[1]}$.

The texture feature of Thangka image is very complex. Thangka painting composition relationship by Thangka religious paintings, is with very strict structure characteristics. In addition, a large number of applications in the Thangka paintings the lightness color gradient, and Thangka colour has a certain level. Therefore, We may start this study mainly from the structural features of Thangka image.

\section{The digital image restoration based on the structure}

Image restoration based on the structure uses the known information around the damaged area in image, determines the direction to be repaired at the diffusion of information spreading within the boundary, and until the damaged area has been restored.

In 2000, Bertalmio put forward such as digital image repair terms explicitly for the first time at SIGGRAPH conference, but also put forward a similar fluid mechanics Navier-Stokes equations of new form of equation ${ }^{[2]}$. It actually is to create a intensity of illumination, such as line as extension direction of image restoration model, and this model is now known as BSCB model. BSCB model does not need to estimate in advance the damage area of each pixel values of pixels, in keeping the illumination, such as line and the boundary Angle at the same time, the image information diffusion in the direction of the illumination, such as line, belongs to the anisotropic diffusion. The algorithm in a narrow area of damage or fracture can get good repairing effect, but due to the nature of the algorithm itself, there is a specific implementation process complex lead to the algorithm and performs slower shortcomings.

In 2001, Chan, etc proposed the premise of the variational (Total Variation, TV) model under Bertalmio and other people's work and BSCB insufficient algorithm ${ }^{[3]}$. It used an eulerian-Lagrangian equation, by minimizing the functional and the anisotropic diffusion equation of energy to accomplish image restoration. TV model can be used to repair the breakage of the smaller area, and can obtain good results. But in a TV model diffusion strength only depends on the intensity of illumination, such as line, and did not consider line intensity of illumination geometry information, such as connectivity. So it is easy to destroy the visual in the process of repair. In 2002, Chan put forward CDD model based on TV model. In the diffusion model of CDD, conduction coefficient depends on the curvature of the illumination line, and not just the intensity of illumination line, and thus obtained the good effect of visual communication, at the same time can be repaired more than TV model area ${ }^{[4]}$.

In 2003, Jia and others put forward a new robust image restoration algorithm, and the image restoration process is divided into two steps: first of all, according to the image texture information of region segmentation, and the second is to use a $\mathrm{N}$ tensor to determine the damage area of the image. This partition method is used to a certain extent, and improve the quality of the precision and quality of image restoration $^{[5]}$. In 2004, Shao Xiaowei and others put forward the adaptive algorithm of a TV 
model. Based on the analysis of the TV model theory, the adaptive method is applied to the structural repair, and the iterative process was optimized. It improved the efficiency of image restoration, and also improved the quality of the repair in a certain extent ${ }^{[6]}$.

In 2005, J.sun and others introduced structure repair concept based on structure-borne ${ }^{[7]}$. Through the use of structural approximate curve first complete structure repair, dividing the whole image for repair again, and this algorithm has a great influence on the solution of the structural deviation. That same year, Andrei and Marcel put forward the repair method based on the edge through calculating the edge of the minimum cost to reconstruct the image ${ }^{[8]}$. Shen and others by using structure information to boot image restoration, in a certain extent, improved the structure after the restoration of blurring effect, and at the same time improve the efficiency of texture synthesis ${ }^{[9]}$.

In 2008, Hao and others proposed the matching algorithm using the structure and texture feature. It repaired the damaged image with the algorithm structure of spread and spread of texture two channels at the same time. The continuity of structure information has a certain improvement, and the repair effect is also more natura ${ }^{[10]}$. In the same year, Wallg and others improved the algorithm for shen and others in $2007^{[11]}$. The image using the structure curve is divided into several sub areas to fill respectively, reduces the texture matching error, and the repair effect has improved a lot. Zhang Fu-mei put forward the CDD model which is an extension of TV mode ${ }^{[12]}$, and considering the geometric characteristics of the image in the process of spreading information curvature. Such not only can handle large damaged area, and the gray level image processing effect is good. But the more obscure boundary is the obvious shortcomings. In order to be able to speed up the speed of image restoration, Zhao Yan-wei and others puts forward a fast image restoration algorithm based on curvature drive repair model ${ }^{[13]}$.

In 2009, Shen and others put forward principle of repair based on maximum a posteriori probability. By using prior knowledge to repair repair to limit the scope, so as to better solve the bad point often appears in remote sensing image and noise phenomenon. After experimental verification, the algorithm by fully considering the characteristics of the visual system is more sensitive to the structural information. For structure is not very complex images were gained satisfactory repairing effect ${ }^{[14]}$.

In 2010, Wei Xin and others proposed image restoration algorithm based on the structure and texture, which is a combination of Poisson equation and block the thoughts of texture synthesis. First of all to the original image is decomposed into structure graph and the texture subgraph, and then according to the features of two independent subgraph respectively to repair. The sub image structure, is repaired based on the Passion equation method. For texture subgraph, adopt texture synthesis method to repair ${ }^{[15]}$.

In 2011, Cui Xue-hong proposed image restoration algorithm of flow linear structure ${ }^{[16]}$. By convection structure of image restoration are analyzed and the research, discussed the tensor diffusion model on the flow structure advantages in image restoration, and extend it to image restoration applications, the structure tensor was used to measure the local structure of the image. Liu Kui put forward image restoration method based on structure tensor, and the structure tensor as the diffusion coefficient of the anisotropic diffusion equation, implemented in different regions have different ways of spreading ${ }^{[17]}$. Gan Ling put forward image restoration algorithm based on the structure and the color information ${ }^{[18]}$. On the basis of Criminisia algorithm, in order to improve the weight to repair known pixels in the image, to join the color information, and used the new matching search methods and the structure of the custom-matching distance weighting criterion for similar block matching.

In 2012, Wu Xiao-jun introduced new priority value and penalty term in the traditional exemplar based inpainting method to improve the performance of it. After combining the linear and curve structural features, They proposed an exemplar and structure based inpainting algorithm for large scale missing regions ${ }^{[19]}$. Yang Xiu-hong presented an image inpainting algorithm based on the weighted fractal under the structure-measurement constraint ${ }^{[20]}$.

In 2013, Li Zhi-dan proposed an adaptive image inpainting algorithm based on patch structure sparsity, in the light of the relationship between the characteristics of damage region and patch 
structure sparsity. According to patch structure sparsity value of the point which had the maximal prority value, the size of patch to be filled, the neighborhood consistence weight and the part-search region size were adaptively confirmed ${ }^{[21]}$.

In 2014, Zhu Xiao-lin and others presented an image restoration algorithm by integrating structure and texture features with reconstruction of significant structure of images ${ }^{[22]}$. In 2015, Sample image dictionary has poor adaptability and simplex valid information, which results in bad image sparse representation. Because of the shortage, Kang Jia-lun proposed a new image inpainting method by characteristics classification learning and patch sparsity propagation ${ }^{[23]}$.

The digital image restoration based on the structure, while considering the correlation between the image features, but there is no combination of image composition, image symbols, decorative pattern, and other forms of concrete structure. Thangka image has its own unique structure characteristic, and broken Thangka image should also be combined with the feature of its own composition digitally restored.

\section{Thangka image inpainting method}

The ancient Tangka image with reflecting Tibet art has been broken by the nature and human with different degree . The part-shed Tangka image is researched as a type of special breaking and analyzed the stability of Hopfield Neural Network . The color clustering segmentation to broken Tangka image by researching Hopfield network model in the HSI color space and using it's memory specialty can be performed. The method can extract effectively Tangka image breaking section ${ }^{[24]}$. But the neural network structure determines the speed of convergence, it is still very slow compared to other clustering methods.

According to the damaged image around, Liu Huaming repairs the image damaged areas with most similar retrieved image area. There is some certain value of the repair technology of Tangka image based on the sample block. Especially it provides a certain degree of technical support to a certain extent. But there are the negative effects especially on the restoration and protection of Tangka image $^{[25]}$. It also brings a certain extent, the negative effects such as repairing image edge uncoordinated and inconsistency direction.

Xiaobao Lu takes Thangka image as research object, and makes some study on the automatic selection of digtal image inpainting algorithms by studying the inpainting algorithms based on PDE and the exemplar-based inpainting algorithms in depth and combining the shape of damaged block, it's adjacent information with the characteristics of these algorithms ${ }^{[26]}$.

Liu Hua-ming proposed that can segment the damaged regions according to the classification of damaged area, aiming at the characteristics of damaged regions in Thangka for missing colors. The edges can be obtained using gray Gradient modules; the regions can be gained through by edges spreading before removing the fake edges. The regions are divided into small regions and large regions, which are handled separately. Small damaged regions can be obtained after removing the fake damaged regions; large regions need a series of processing, removing fake damaged regions, extracting the large regions, remaining complex regions; the tiny damaged regions and damaged holes regions are extracted from the complex regions, they are combined, dilated, the damaged regions are obtain after removing fake damaged regions. The small damaged regions, large damaged regions and the damaged regions in the complex regions are combined into together, the damaged regions are segmented in the whole image ${ }^{[27]}$. But this method on complex damaged areas accurately segmentation still need further improvement.

Liu Zhong-min proposed a new image inpainting method based on morphological component analysis. The proposed algorithm utilizes p-Laplace operator in the information spread not only along the edge direction, but also in gradient direction, which not only preserves edge, but also avoids staircase in the smooth area, and at the same time, the result is also less sensitive to noise ${ }^{[28]}$. 


\section{Problems and prospects}

Now, many domestic and foreign scholars put forward to a variety of highly effective repair methods in the field of digital image restoration models and algorithms. But There are very few research on Tangka image inpainting which uses these digital image restoration techniques .

The existing research mainly from the image retrieval, image segmentation and morphological component analysis Angle. Although this has achieved certain success in repairing effect, but still make use of the general features of image repair. And Thangka painting composition relationship by Thangka religious paintings, the frame which can find the beauty of symmetry and balance, contrast and unity of beauty, the beauty of rhythm and rhyme, there is no relevant research work given in the process of image restoration.

The basic composition method of Thangka is composition of one point, composition of two points, and three line composition. Moreover, there is a certain level in the color of Thangka image. How to structure features of Thangka as a research focus, how to combine Thangka painting composition, image symbols, decorative pattern, and other forms of concrete structure, how to use the structure of digital Thangka spread way, to disperse the literature of Thangka, gallery, related information, such as digital processing, through integration of resources, to realize "Digital Thangka ", will be the research focus on topics.

\section{Acknowledgements}

This paper is supported in part by The National Natural Science Foundation of China (No. 61440021), "Chunhui Plan" Project of Chinese Ministry of Education(No. Z2014004), Science and Technology Project in Qinghai Province(No. 2015-ZJ-725).

\section{References}

[1] Zhang Hong-ying, Peng Qi-cong. A Survey on Digital Image Inpainting[J]. Journal of Image and Graphics,2007,12(1):1-10.

[2] M. Bertalmio, G. Sapiro, V. Caselle et al. Image Inpainting[C].New York: ACM SIGGRAPH, 2000: 417-424.

[3] T.Chan,J.Shen. Non-Texture Inpainting by Curvature-Driven Diffusions(CDD)[J]. Journal of Visual Communication and Image Representation, 2001, 12(4): 436-449.

[4] T.Chan,J.Shen. Mathematical Models for Local Non-Texture Inpaintings[J].SIAM Journal of Applied Mathematics, 2002, 62(3):1019-1043.

[5] J.Jia,C.Tang.Image Repairing:Robust Image Synthesis by Adaptive ND Tensor Voting[C]. Madison: IEEE Computer Society Conference on Computer Vision and Pattern Recognition, 2003: 643-650.

[6] Shao Xiao-wei,Liu Zheng-kai, Song Bi. An Adaptive Image Inpainting Approach Based on TV Model[J]. Jounal of Circuits and System,2004,9(2):113-117.

[7] J.Sun,L.Yuan,J.Y.Jia,et al.Image Completion with Strcture Propagation[J]. ACM Transactions on Graphics,2005,24(3):861-868.

[8] A.Rares,M.J.T.Reinders,J.Biemond. Edge-Based Image Restoration[J].IEEE Transaetionson Image Processing,2005,14(10):1454-1468. 
[9] Shen Minfen,Li Bin.Structure and texture image inpainting based on region segmentation. Proceedings of IEEE International Conference on Acoustics,Speech and Signal Processing.Hawaii,2007:701-704.

[10] G.Hao,O.Nobutaka,and S.Shiqeki.A Structure-Synthesis Image Inpainting Algorithm Based on Morphological Erosion Operation[J]. Image and Signal Proeessing,2008,5(3):530-535.

[11] Wang Minqin,Han Guoqiang,Tu Yongqiu.Edge-based image completing guided by region segmentation[J]. Proceeding of ISECS International Colloquium on Computing,2008:152-156.

[12] Zhang Fu-mei. Color image inpainting based on total variation model[J]. Computer Applications, 2008, 28(4):997-999.

[13] Zhao Yan-wei, Li Xiang-lin. A Rapid Image Inpainting Algorithm Basedon CDD Model[J]. Computer Simulation,25(10), pp.223-227,2008.

[14] H.F.Shen,L.P.Zhang.A MAP-Based Algorithm for Destriping and Inpainting of Remotely Sensed Images[J]. IEEE Trans.Geosci.Remote Sens.,May2009,5(47):1492-1502.

[15] Wei Xin, Jiang Hua-wei. Study on Inpainting Algorithm Based on Image Structure and Texture[J]. Computer Technology and Developent,20(9), pp.90-93,2010.

[16] Cui Xuehong.Flow-like textures with line-like structures images inpainting by improved tensor diffusion model[J]. Computer Engineering and Applications,27(47),pp.206-209,2011.

[17] Liu Kui,Su Ben-yue , Zhao Xiao-jing. Image inpainting method based on structure tensor[J]. Journal of Computer Applications,31(10),pp.2711-2713,2011.

[18] Gan Ling, Zhang We, Liu Guo-qing. Image Inpainting Algorithm Based on the Structure and Color Information[J]. Computer Simulation 28(2),pp.329-332,2011.

[19] Wu Xiao-jun,Li Gong-qing. Large Scale Image Inpainting Based on Exemplar and Structure Information. Acta Electronica Sinica, 40(8),pp.1509-1514,2013.

[20] Yang Xiu-hong , Guo Bao-long, Yan Chunma. Image inpainting algorithm based on the weighted fractal under the structure-measurement constraint[J]. Journal of Xidian University, 39(6), pp.100-108,2012.

[21] Li Zhi-dan, He Hong-jie etc. Adaptive Image Inpainting Algorithm Based on Patch Structure Sparsity. Acta Electroncia Sinica,3(3), pp.549-554,2013.

[22] Zhu Xiao-lin, Chen Xiao-dong and etc. An Image Restoration Algorithm Based on Structure and Texture Synthesis with Reconstruction of Significant Structure of Images[J]. Journal of Graphics,35(3):336-342.

[23] WANG Xiao-dong, WANG Weilan, TANG Shixi. Tangka Image Breaking Section Extraction Based on Hopfield Neural Network. Science Technology and Engineering, 2006, pp.706-717.

[24] Kang Jia-lun, Tang Xiang-hong etc. Image Inpainting by Characteristic Classification Learning and Patch Sparsity

Propagation[J]. Journal of Computer-Aided Design \& Computer Graphics,27(5):864-872

[25]Liu Hua-ming, Tang Shixi, Xie Hui. Exemplar-based inpainting algorithm for broken Tangka image. Fu Jian Computer, 2007, pp .15-16.

[26] Lu Xiao-bao, Research on Tangka Image Inpainting of Comprehensive Partial Differential Equations[M], Northwest University for Nationalities, 2011. 
[27] Liu Hua-ming, BI Xue-hui. Damaged regions segmentation on Thangka for missing colors. Computer Engineering andApplications, 2013, 49(4),pp.219-223, 2013.

[28] Liu Zhong-min, Hu Wen-jin, Li Zhan-ming. An Algorithm for Tangka Image Inpainting Based on Morphological Component Analysis. Computer Engineering,40(1),pp.250-253,2014. 